\title{
Eating and Psychological Profiles of Women with Higher Depressive Symptoms Who Are Trying to Lose Weight
}

\author{
C. Bégin, S. De Grandpré, and M.-P. Gagnon-Girouard \\ School of Psychology, Laval University, Quebec City, QC, Canada G1V 0A6 \\ Correspondence should be addressed to C. Bégin, catherine.begin@psy.ulaval.ca \\ Received 5 December 2011; Revised 23 April 2012; Accepted 15 June 2012 \\ Academic Editor: Jutta Mata
}

Copyright () 2012 C. Bégin et al. This is an open access article distributed under the Creative Commons Attribution License, which permits unrestricted use, distribution, and reproduction in any medium, provided the original work is properly cited.

The aim of this study was to determine whether women with higher depressive symptoms differed from women with lower depressive symptoms on early weight-loss, eating behaviors and psychological profiles. Among a sample of 45 overweight/obese women who had undertaken a self-initiated weight-loss attempt, two groups were formed based on scores from the Beck Depression Inventory (BDI-II), one with lower depressive symptoms (BDI-II $<10 ; n=21$ ) and one with higher depressive symptoms (BDI $\geq 10 ; n=24)$. Even if some women in the higher depressive symptom group did not reach the clinical cutoff for depression $(\mathrm{BDI}=14)$, this group tended to lose less weight in the first two months of their weight-loss attempt and to show a more disturbed eating and psychological profile compared to the group with lower depressive symptoms. In addition, among women with higher depressive symptoms, eating and psychological variables were systematically related to one another whereas these variables were not related among the other group. Results highlight the relevance of considering the presence of depressive symptoms as a marker of clinical severity among the overweight/obese population, and suggest that the BDI-II could be an interesting screening instrument to identify this particular subgroup.

\section{Introduction}

Obesity jeopardizes public health worldwide [1]. Despite the numerous weight-loss programs available, prevalence rates of obesity and overweight relentlessly increase, which suggests that, as many as they are, current weight-loss programs do not succeed in reducing, or even stabilizing, the proportion of individuals who are overweight or obese. One potential explanation might be that most individuals are not able to achieve or maintain weight-loss recommendations in the long term. Individual characteristics could account for these difficulties to regulate body weight. In fact, overweight/obese individuals seem to form a particularly heterogeneous population divided into specific subgroups that can be characterized by distinctive psychological features [2-4].

In particular, women suffer more from their weight problems than men and report more weight preoccupation and body dissatisfaction, regardless of their age, ethnic group, education level, and body mass index (BMI) [3, 5-7]. Women are also overrepresented in weight-loss programs, where 85 to $90 \%$ of participants are women [8-11]. In weight-loss studies, obese women also show greater distress than men; for example, they generally show more depressive symptoms and are more likely to report repetitive and unsuccessful dieting in the past $[12,13]$. These considerations suggest that women represent an at-risk subgroup of obese individuals.

Another subgroup of obese individuals that may present distinctive features leading to strenuous weight regulation is the one characterized by a higher level of depressive symptoms. Overweight/obese women seem to be more vulnerable to depressive symptoms when compared to overweight/obese men and to normal-weight women [14]. Yet, all obese women are not systematically characterized by depressive symptoms, and the correlation between obesity and depression is significant but weak. In fact, while some studies reported a positive association [15-20] as well as a longitudinal bidirectional relationship between these two concepts [21], other studies reported a weak or no association at all $[3,22,23]$. These findings may suggest that, while some overweight/obese women are asymptomatic, another subgroup of women does show depressive symptoms, which may interact with other factors affecting eating behaviors and weight-loss efforts. Recently, several research groups have 
observed the eating and the psychological differences between these two groups.

Werrij and her colleagues [24] studied this phenomenon among a sample of 166 women seeking treatment at a weight-loss center. Half of the sample was categorized as the higher depressive symptoms group, whereas the other half was identified as the lower depressive symptoms group on the basis of the Beck Depression Inventory score (median = 10). Women with higher depressive symptoms had higher weight, shape, and eating concerns as well as lower selfesteem. They also reported higher dietary restraint and greater binge eating. However, in this sample, women with higher depressive symptoms presented a higher BMI than the other women, which could account for the observed differences.

Subsequent studies have corroborated these results. Cluster analyses led to the identification of a higher negative affect group and a lower negative affect group among a sample of obese/overweight women from the community $[25,26]$. Women with higher negative affect were younger and reported higher weight, shape and eating concerns, more binge eating, and lower self-esteem. Moreover, when exposed to negative mood induction, only women in the higher negative affect group presented disinhibited eating. These differences between the two groups could not be attributed to body weight, since groups had similar BMI.

Another study conducted among a sample of 156 overweight/obese weight-preoccupied women, involved in a Health-at-Every-Size program [27] replicated previous findings, and went further by showing that women with higher depressive symptoms presented some particular personality traits, notably high neuroticism and low agreeableness as well as problematic coping strategies, such as more emotionoriented and avoidance strategies, and less task-oriented strategies. Again, these differences could not be attributed to body weight since there was no difference in BMI between groups. However, in this study, numerous exclusion criteria were used to identify eligible participants (e.g., no eating disorders, stable weight for two months, no clinical depression, etc.), which may limit the representativeness of the sample. Thus, taken altogether, these results suggest that women who present higher depressive symptoms may exhibit distinctive features that could impede weight-loss efforts.

Other studies support the hypothesis that depressive symptoms could be related to less successful weight-loss outcomes [13, 28-31]. Indeed, there is a significant, though weak, negative correlation between weight-loss and depressive symptoms $(r=-0.15 ; P<0.01)[13]$. In the same order of idea, it has been shown that women with higher depressive symptoms are less likely to complete a weight-loss program or to succeed in losing weight [29-51]. Fabricatore and his colleagues [29] suggested that demands which are inherent to weight-loss programs may be too challenging for women with significant depressive symptoms.

Up until now, no study has dichotomized women based on depressive symptoms to observe their weight-loss outcomes. Moreover, most weight-loss studies have been conducted using well-controlled, randomized protocols, and structured weight-loss programs. Yet, some authors suggest that outcomes from controlled studies using structured weight-loss programs may differ markedly from what happens to women who try to lose weight on their own. In fact, these women are likely to be less rigorous and to lose smaller amount of body weight $[13,32,33]$.

The study aimed at deepening the understanding of subgroups of overweight/obese women separated on the basis of depressive symptoms. Specifically, the objective of this study was to determine, in a naturalistic study, whether women with higher depressive symptoms differed from women with lower depressive symptoms on early weight-loss outcomes as well as on eating and psychological profiles, and to examine the links between all these variables among the different groups. We expected that higher depressive symptoms women would lose less weight compared to women with lower depressive symptoms and that they would present more problematic eating behaviors and less positive psychological features.

\section{Method}

2.1. Participants. Forty-five women who corresponded to inclusion criteria (being premenopausal, i.e., reporting a regular menstrual cycle for the last three months, having a BMI of $25 \mathrm{~kg} / \mathrm{m}^{2}$ or more, and having initiated a weight-loss attempt in the last week) completed baseline measures (questionnaires and physical measurements) (week 1). Thirty-six women came to the subsequent weighing visits (week 2 to week 8). Among women who dropped out of the study ( $n=$ 9), four moved away, two decided to stop for personal reasons and three could not be reached by the research team during the course of the study.

Women were aged between 21 and 56 years $\left(M_{\mathrm{age}}=\right.$ $37.6 \pm 9.6)$. Twenty-four $(53.3 \%)$ were in a relationship (married or in couple) while $21(46.6 \%)$ were not (i.e., single, separated, or divorced). All women completed a high-school diploma, and $26(57.3 \%)$ completed a university degree. At baseline, mean BMI was $30.58 \pm 4.83\left(M_{\mathrm{BMI}}\right.$ for the lower depressive symptoms group $=31.05 \pm 4.79 ; M_{\mathrm{BMI}}$ for the higher depressive symptoms group $=30.16 \pm 4.93$ ). All women had initiated a weight-loss attempt one week or less before the baseline visit. Weight-loss methods ranged from reducing portions or trying to exercise more by themselves $(n=29)$, enrolling in commercial weight-loss programs with strict eating rules such as Weight Watchers and Minçavi programs $(n=13)$, or reading self-help books $(n=3)$. There was no significant difference between groups concerning the methods that were used to lose weight (Pearson chisquares were not significant, $P=0.688$ to 0.905 ). Only six women $(13.3 \%)$ had never tried to lose weight before. Women had become preoccupied with their weight around the end of their adolescence or at the beginning of adulthood $\left(M_{\text {age of first preoccupations }}=17.69 \pm 7.71\right.$ years $)$.

2.2. Procedure. For recruitment, advertisements were placed in commercial weight-loss and fitness centers, as well as in different places on the Laval University campus. In addition, 
emails were sent to all students and staff of the university campus.

All participants were met at baseline (week 1), were told about the aims and procedures of the study, and were asked to read and sign the consent form approved by the ethics committee of the university. Subsequently, detailed questions regarding their current weight-loss attempt were asked. Participants were then measured and weighed. They were asked to complete self-reported questionnaires at baseline only. Participants came back once a week for the first six weeks (week 2 to 6), and once, two weeks later (week 8), to be weighed. Weekly weighing for the first six weeks was proposed in order to carefully monitor the early weightloss process as well as to improve women's motivation to participate in the study.

\subsection{Measures}

2.3.1. Physical Measurements. Height and weight were measured and BMI was calculated based on standard procedures [34].

2.3.2. Sociodemographic and Weight-Loss-Related Information. Sociodemographic information such as age, education, and marital status was collected. Age at first weight concerns and first weight-loss attempt, number of previous weightloss attempts, and description of current weight-loss method were included in the sociodemographic questionnaire.

2.3.3. Depressive Symptoms. The Beck Depression Inventory (BDI-II [35]), a 21-item questionnaire, was used to measure depressive symptoms. The BDI-II presents a good internal consistency ( $\alpha=0.92$ in a nonpsychiatric population) and a good test-retest reliability (one week: $r=0.93$ ) [35]. Severity of depressive symptoms increases with scores $(0-13=$ minimal symptoms; $14-19=$ mild depression; $20-28=$ mod erate depression; 29 or more $=$ severe depression) .

2.3.4. Eating Behaviors. The Three-Factor-Eating Questionnaire (TFEQ [36]), a well-known 51-item questionnaire divided in three subscales (Cognitive dietary restraint, Disinhibition, and Susceptibility to hunger), was used to measure eating behaviors. The internal consistency is good for the three factors of the questionnaire ( $a=0.92,0.91$, and 0.85 ) [36].

2.3.5. Motivation. The Global Motivation Scale (GMS [37]), a 24-item questionnaire, was used to evaluate six different types of motivation (intrinsic, integrated, identified, introjected, external motivation, and amotivation), based on the Self-Determination Theory of Deci and Ryan [38]. Reliability and validity of the GMS have been established by five independent studies [37]. The Regulation of Eating Behaviors Scale (REBS [39]) was used to evaluate motivation to regulate eating behaviors specifically. The REBS internal consistency is good ( $a$ between 0.72 and 0.95 depending of the subscale [39]).
2.3.6. Quality of Life. The Impact of Weight on Quality of Life Questionnaire (IWQOL-Lite [40]) is a 31-item questionnaire that measures quality of life related to weight. The IWQOL-Lite shows high internal consistency $(\alpha=0.96$ for total score) and satisfying convergent and discriminant validity $[41]$.

2.3.7. Body Esteem. The Body-Esteem Scale (BES [42]), a 23item questionnaire used to measure the level of satisfaction of individuals toward their body image, is divided in three subscales: Appearance, Weight, and Attribution. Very high internal consistency coefficients $(\alpha=0.92,0.81$, and 0.94 ) as well as good temporal stability have been demonstrated for each scale ( $\alpha=0.89,0.92$, and 0.83$)$ [42].

2.3.8. Self-Esteem. The Rosenberg Self-Esteem Scale (RSES [43]) was used to measure self-esteem. The 10-item questionnaire measures the global self-concept and the feeling of personal worth. Internal consistency varies from 0.83 [44] to 0.99 [45] and test-retest reliability is 0.82 [46].

2.3.9. Personality. The Temperament and Character Inventory (TCI [47]) was used to measure pathological and nonpathological dimensions of personality in terms of Temperament (Harm avoidance, Novelty seeking, Reward Dependence, and Persistence) and Character (Self-Determination, Cooperativeness, and Self-Transcendence). The TCI shows a good internal consistency ( $a>0.80$ depending of subscales) [48].

2.4. Statistical Analyses. First, descriptive analyses were computed for the whole sample. Means, standard deviations and Pearson's correlations between the different variables were computed with the SPSS software (version 13.0). As suggested by Werrij and her colleagues [24], two groups of women were formed based on the BDI score $($ median $=10)$; a first group, with a BDI score of less than $10(n=21)$ and a second group, with a BDI score of 10 or more $(n=$ 24). To test differences between groups, univariate analyses of variance were computed for the following dependent variables: baseline BMI, number of previous weight loss attempts, percentage of weight loss, and impact of weight on quality of life. Multivariate analyses of variance were computed for age variables (model $1=$ current age, age at first weight concerns, age at first weight-loss attempt), eating behaviors (model $2=$ cognitive dietary restraint, disinhibition, susceptibility to hunger), body- and self-esteem (model 3 = self-esteem, body esteem related to appearance, weight and attribution), personality (model $4=$ novelty seeking, harm avoidance, reward dependence, persistence, self-determination, cooperativeness, self-transcendence), motivation to regulate eating behaviors (model $5=$ intrinsic, integrated, identified, introjected, external motivation and amotivation), and global motivation (model $6=$ intrinsic, identified, introjected, external motivation, and amotivation). Bonferroni correction was used to correct for the number of comparisons, $(\alpha=0.05 / 10$ tests $=0.005)$, meaning that results were considered significant if $P<0.005$. 
TABLE 1: Differences between groups for age-, weight, and eating-related variables.

\begin{tabular}{|c|c|c|c|c|}
\hline Variables & $\begin{array}{l}\text { Lower depressive SX }(n=21) \\
\qquad M(\mathrm{ET})\end{array}$ & $\begin{array}{l}\text { Higher depressive SX }(n=24) \\
\qquad M(\mathrm{ET})\end{array}$ & Anova MANOVA & $P$ \\
\hline Baseline BMI & $31.05(4.79)$ & $30.16(4.93)$ & $F(1,43)=0.37$ & 0.548 \\
\hline Age $^{\mathrm{a}}$ & $41.56(8.82)$ & $35.50(9.38)$ & $F(1,43)=4.07$ & 0.050 \\
\hline Age of first weight concerns ${ }^{a}$ & $20.56(7.85)$ & $14.45(6.14)$ & $F(1,40)=7.25$ & 0.011 \\
\hline Age of first weight-loss attempts ${ }^{a}$ & $24.69(9.23)$ & $17.59(6.06)$ & $F(1,38)=8.20$ & 0.007 \\
\hline \multicolumn{5}{|l|}{ 2-month weight loss } \\
\hline (in \%) & $2.74(2.43)$ & $1.13(1.66)$ & \multirow{2}{*}{$F(1,34)=5.41$} & \multirow{2}{*}{0.026} \\
\hline (in pounds) & $5.05(5.01)$ & $1.93(3.00)$ & & \\
\hline Number of previous weight-loss attempts & $5.41(4.77)$ & $8.04(10.43)$ & $F(1,38)=0.79$ & 0.380 \\
\hline TFEQ-Restraint $^{\mathrm{b}}$ & $10.19(3.23)$ & $9.67(4.87)$ & $F(1,43)=0.18$ & 0.678 \\
\hline TFEQ-Disinhibition $^{\mathrm{b}}$ & $8.10(3.49)$ & $11.21(2.75)$ & $F(1,43)=11.17$ & $0.002 *$ \\
\hline TFEQ-Hunger ${ }^{\mathrm{b}}$ & $3.52(3.08)$ & $8.58(3.54)$ & $F(1,43)=25.84$ & $<0.001^{*}$ \\
\hline
\end{tabular}

${ }^{*} P<0.005$ (Bonferroni correction); ${ }^{\mathrm{a}}$ MANOVA 1 ; ${ }^{\mathrm{b}}$ MANOVA 2.

TABLE 2: Differences between groups for psychological variables.

\begin{tabular}{|c|c|c|c|c|}
\hline Variables & $\begin{array}{l}\text { Lower depressive SX }(n=21) \\
\qquad M(\mathrm{ET})\end{array}$ & $\begin{array}{l}\text { Higher depressive SX }(n=24) \\
\qquad M(\mathrm{ET})\end{array}$ & Anova MANOVA & $P$ \\
\hline BES-Appearance ${ }^{c}$ & $1.69(0.65)$ & $1.10(0.65)$ & $F(1,43)=9.27$ & $0.004 *$ \\
\hline BES-Weight ${ }^{c}$ & $1.21(0.63)$ & $0.69(0.50)$ & $F(1,43)=9.44$ & $0.004^{*}$ \\
\hline BES-Attribution ${ }^{c}$ & $1.99(0.52)$ & $1.68(0.57)$ & $F(1,43)=3.58$ & 0.065 \\
\hline RSES $^{c}$ & $34.52(4.38)$ & $27.38(6.86)$ & $F(1,43)=16.80$ & $<0.001^{*}$ \\
\hline IWQL & $79.38(10.88)$ & $68.34(16.00)$ & $F(1,43)=7.11$ & 0.011 \\
\hline TCI-Novelty Seeking ${ }^{\mathrm{d}}$ & $21.14(4.27)$ & $21.29(7.54)$ & $F(1,43)=0.06$ & 0.937 \\
\hline TCI-Harm Avoidance ${ }^{\mathrm{d}}$ & $13.19(4.56)$ & $16.92(7.98)$ & $F(1,43)=3.56$ & 0.066 \\
\hline TCI-Reward Dependence ${ }^{\mathrm{d}}$ & $15.19(3.78)$ & $15.21(4.27)$ & $F(1,43)=0.00$ & 0.988 \\
\hline TCI-Persistence ${ }^{\mathrm{d}}$ & $5.00(2.02)$ & $5.54(2.15)$ & $F(1,43)=0.75$ & 0.391 \\
\hline TCI-Self-Determination ${ }^{\mathrm{d}}$ & $35.48(3.67)$ & $23.88(8.89)$ & $F(1,43)=31.08$ & $<0.001^{*}$ \\
\hline TCI-Cooperativeness ${ }^{\mathrm{d}}$ & $37.33(2.54)$ & $32.04(5.93)$ & $F(1,43)=14.38$ & $<0.001^{*}$ \\
\hline TCI-Self-Transcendence ${ }^{\mathrm{d}}$ & $11.29(6.24)$ & $12.08(6.95)$ & $F(1,43)=0.16$ & 0.689 \\
\hline
\end{tabular}

${ }^{*} P<0.005$ (Bonferroni correction); ${ }^{\mathrm{C}}$ MANOVA 3; ${ }^{\mathrm{d}}$ MANOVA 4.

Effect sizes (Cohen's $d$ ) were also calculated; an effect size of 0.3 was considered a small effect, 0.5 , a medium effect, and 0.8 , a large effect [49]. The number of previous weight-loss attempts was logged 10 transformed to correct for skewness.

\section{Results}

Means and standard deviations for each group (higher versus lower depressive symptoms) concerning age-, weight ${ }^{\sim}$, and eating-related variables, as well as psychological and motivational variables are presented, respectively, in Tables 1,2 , and 3 . The mean BDI score for the overall sample was $11.29 \pm 1.58$. For the lower depressive symptoms group, the mean BDI was $3.62 \pm 0.58$, ranging from 0 to 8 , whereas for the higher depressive symptoms group, the mean BDI was $18 \pm 2$ ranging from 10 to 50 . In accordance, even though this group's mean score was above the clinical cut-off of 14 for mild depression, some women of this group did not reach this clinical cut-off (score between 10-13; $n=12$ ), meaning that the group cannot be labelled as "depressed." Among other women belonging to the high depressive symptoms group, four presented mild depression (score between 14 and 19), five reported moderate depression (score between 20 and 28), and three were severely depressed (score over 29).

First, participants from both groups did not differ regarding baseline BMI, $F(1,43)=0.37, P=0.548$, meaning that differences between groups cannot be attributed to body weight. Second, women tended to be different on age variables, $F(1,43)=3.16, P=0.037$. Women with higher depressive symptoms tended to be younger, $F(1,43)=4.07$, $P=0.050$, to report having made their first weight-loss attempt earlier, $F(1,38)=8.20, P=0.007$, and having started earlier to be preoccupied with their weight $F(1,38)=$ $7.25, P=0.011$, compared to women presenting lower depressive symptoms. Even though these differences were not significant with the Bonferroni correction, effect sizes were $0.67,0.89$, and 0.87 , which correspond to moderate to large effects. In addition, women' current age was significantly related to their age at first weight concerns, $r=0.33, P=$ 0.035 , which means that the younger women started to be preoccupied with their weight, the younger they were when 
TABLE 3: Difference between groups for motivational variables.

\begin{tabular}{|c|c|c|c|c|}
\hline Variables & $\begin{array}{l}\text { Lower depressive SX }(n=21) \\
\qquad M(\mathrm{SD})\end{array}$ & $\begin{array}{l}\text { Higher depressive SX }(n=24) \\
\qquad M(\mathrm{SD})\end{array}$ & MANOVA & $P$ \\
\hline REBS-Intrinsic ${ }^{\mathrm{e}}$ & $5.15(1.44)$ & $4.34(1.44)$ & $F(1,43)=3.58$ & 0.065 \\
\hline REBS-Integrated ${ }^{\mathrm{e}}$ & $5.33(1.22)$ & $4.24(1.51)$ & $F(1,43)=7.03$ & 0.011 \\
\hline REBS_-Identified ${ }^{\mathrm{e}}$ & $6.36(0.73)$ & $5.88(1.09)$ & $F(1,43)=2.95$ & 0.093 \\
\hline REBS-Introjected ${ }^{\mathrm{e}}$ & $3.02(1.03)$ & $3.98(1.71)$ & $F(1,43)=4.98$ & 0.031 \\
\hline REBS-External ${ }^{\mathrm{e}}$ & $1.54(1.02)$ & $2.40(1.86)$ & $F(1,43)=3.54$ & 0.066 \\
\hline REBS-Amotivation ${ }^{\mathrm{e}}$ & $1.17(0.37)$ & $1.65(0.91)$ & $F(1,43)=5.08$ & 0.029 \\
\hline GMS_-Intrinsic ${ }^{f}$ & $5.64(1.15)$ & $5.14(1.19)$ & $F(1,43)=2.01$ & 0.164 \\
\hline GMS_-Identified $^{f}$ & $5.51(0.94)$ & $5.16(0.94)$ & $F(1,43)=1.50$ & 0.228 \\
\hline GMS_-Introjected $^{\mathrm{f}}$ & $3.43(1.31)$ & $4.10(1.26)$ & $F(1,43)=2.98$ & 0.092 \\
\hline GMS_External $^{\mathrm{f}}$ & $3.43(1.31)$ & $4.10(1.26)$ & $F(1,43)=2.98$ & 0.092 \\
\hline GMS-Amotivation $^{\mathrm{f}}$ & $1.59(0.61)$ & $2.20(1.39)$ & $F(1,43)=3.36$ & 0.074 \\
\hline
\end{tabular}

${ }^{*} P<0.005$ (Bonferroni correction); ${ }^{\mathrm{e}}$ MANOVA 5; ${ }^{\mathrm{f}}$ MANOVA 6.

they enrolled in the study. In addition, age at first weight concerns and age at first weight-loss attempt were strongly related for both groups (lower depressive symptoms group, $r=0.73, P=0.001$; higher depressive symptoms group, $r=0.86, P<0.001$ ), which means that being preoccupied earlier with body weight was related to an early weight loss attempt.

Results also showed that, following a two-month selfinitiated weight-loss attempt, women with higher depressive symptoms tended to lose less weight than the other women, $F(1,34)=5.41, P=0.026$. Even though the difference was not significant after the Bonferroni correction, the effect size was 0.77 , which is a moderate effect. However, women with higher depressive symptoms did not differ regarding the number of previous weight-loss attempts, $F(1,38)=0.79$, $P=0.380$.

Women with higher depressive symptoms also showed significantly more problematic eating behaviors, $F(1,43)=$ $8.71, P<0.001$, with higher scores on disinhibition, $F(1,43)=11.17, P=0.002$, and susceptibility to hunger, $F(1,43)=25.84, P<0.001$. Effect sizes for eating behaviors were large, respectively, of 0.99 and 1.52. From a psychological standpoint, women with higher depressive symptoms tended to report a significantly more negative selfperception, $F(1,43)=4.25, P=0.006$, with lower scores regarding self-esteem (RSEQ), $F(1,43)=16.80, P<0.001$, body esteem (BES) related to appearance, $F(1,43)=9.27$, $P=0.004$, and body esteem related to body weight, $F(1,43)=9.44, P=0.004$. Effect sizes for these differences were, respectively, of 1.24 (Self-esteem), 0.91 (Body esteemappearance), 0.92 (Body esteem-weight), which correspond to large effects. They also tended to present lower quality of life (IWQL-Q), $F(1,43)=7.11, P=0.011$. Even though this difference was not significant with the Bonferroni correction, the effect size was 0.81 , which corresponds to a large effect.

Concerning personality, significant differences between groups were observed, $F(1,43)=6.05, P<0.001$, women with higher depressive symptoms reporting significantly lower Self-Determination, $F(1,43)=31.08, P<0.001$, and Cooperativeness, $F(1,43)=14.38, P<0.001$, on the TCI, which means that women with higher depressive symptoms reported being less confident in their power to influence their own life and less prone to accept and understand others, and to be empathic and altruistic. Effect sizes for these differences were, respectively, of 1.71 and 1.16, which correspond to large effects.

In general, results showed that there was no significant difference concerning global motivation, $F(1,43)=1.47$, $P=0.229$, and marginally significant differences between groups regarding motivation to regulate eating behaviors (REBS), $F(1,43)=2.12, P=0.074$. Women with higher depressive symptoms tended to show more introjected motivation to regulate their eating behaviors than women with lower depressive symptoms, $F(2,30)=4.98, P=0.031$, meaning that they may put on themselves some pressure to adopt particular eating behaviors in order to avoid feeling guilty and ashamed. Women with higher depressive symptoms also tended to report more amotivation, $F(1,43)=$ $5.08, P=0.029$, which suggests that they may feel a lack of control over their own eating behaviors. These women also tended to report lower integrated motivation, $F(1,43)=$ 7.03, $P=0.011$, meaning that they may engage in eating behaviors without having integrated the importance and value of these behaviors. Effect sizes for these differences were, respectively, of $0.68,0.69$, and 0.79 , which correspond to moderate effects.

Finally, correlational analyses were computed separately for each group (Table 4). In general, in the higher depressive symptoms group, variables were more closely associated with one another than in the lower depressive symptoms group. For example, among women with higher depressive symptoms, self-esteem, body esteem related to weight, body esteem related to appearance, quality of life, and susceptibility to hunger were systematically related to depressive symptoms and correlated to one another, whereas these associations were clearly not significant among the lower depressive symptoms group. Likewise, among women with higher depressive symptoms, Self-Determination (the feeling to have an influence on one's life) was strongly related to disinhibition, susceptibility to hunger, self-esteem, and 


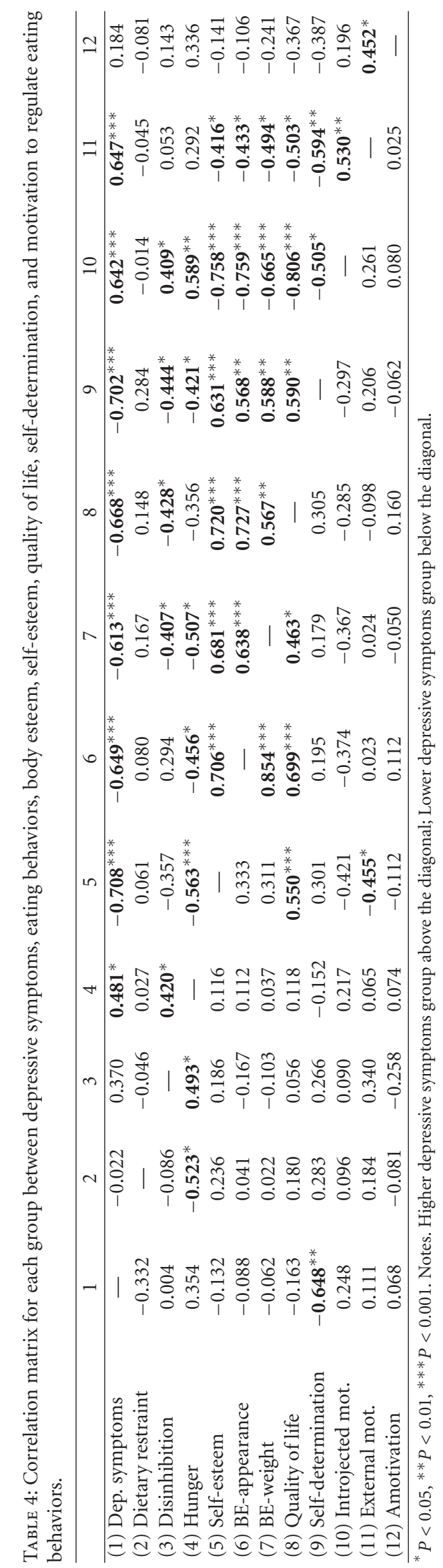


body esteem related to weight and appearance as well as to quality of life, whereas these associations were again not significant among women who are less symptomatic. As demonstrated in Table 4, several other associations followed the same pattern.

\section{Discussion}

The objective of the present study was to compare two groups of overweight/obese women formed on the basis of depressive symptoms on initial weight-loss outcomes as well as on eating and psychological profiles. Several results from the present study confirmed what has been previously found $[25,26]$. Women with higher depressive symptoms were more prone to lose control over eating (disinhibition), were more inclined to eat in the presence of perceptions or feelings of hunger (susceptibility to hunger), reported a lower general feeling of self-worth (self-esteem), were less satisfied with their appearance and their weight (body esteem), and presented a lower quality of life related to weight $[24,26,27]$ and thus, despite the fact that they did not differ with regard to body weight compared to women with lower depressive symptoms. For women with higher depressive symptoms, other factors, besides body weight, may influence body esteem and quality of life; among this group, quality of life and body esteem related to weight were both significantly associated with depressive symptoms and disinhibition, which suggests that negative affect toward one's self and disinhibited eating associated to a feeling of loss of control could hinder body esteem and perceived quality of life, regardless of weight.

Results also suggested that, compared to women with lower depressive symptoms, those with higher depressive symptoms were younger, have been concerned earlier by their weight (i.e., from the age of 14 for women with higher depressive symptoms and from the age of 21 for women with lower depressive symptoms), and have undertook weightloss attempts earlier (i.e., almost seven years before, from the age of 17). In accordance, for the overall sample, current age was related to the age at first weight concerns and to the age of the first weight-loss attempt, which could explain why other studies have also found a younger age among participants from the higher depressive symptoms group. However, groups did not significantly differ regarding the number of previous weight-loss attempts, as was evidenced in previous studies [50]. This could be attributable to the small sample size and the fact that most women from both groups had undertaken many weight-loss attempts at the time of their participation in the study.

In addition, women with higher depressive symptoms tended to lose less weight than women from the other group during the two first months of a naturalistic weight-loss attempt. To our knowledge, this is the first study that examined whether subgroups separated on the basis of depressive symptoms could differ on weight-loss outcomes following a self-initiated weight-loss process. In the literature, it is suggested that depressive symptoms, even if of subclinical severity, may negatively influence weight loss [12, 28-31, 51], but up until now, no study had yet documented this issue. Since it is well established that early weight loss is a significant predictor of long-term weight loss [30], we may hypothesize that women from our higher depressive symptoms group may experience more difficulties in the long-term pursuit of their weight lost. In fact, their initial failure may portend their long-term failure.

One potential explanation for this difference in terms of early weight loss is that, among women with higher depressive symptoms, all variables such as eating behaviors, self-esteem, body esteem, and quality of life were closely related to one another, whereas it was not the case among less depressed women. Results suggest that among women with higher depressive symptoms, these variables interacted to form a negative spiral, which worsens the overall picture and creates a vicious circle of distress and negative self-perception that may subsequently hinder weight-loss efforts.

In addition, women with higher depressive symptoms tended to endorse less integrated motivation, more introjected motivation, and more amotivation to regulate their eating behaviors. According to Pelletier, Dion, and colleagues [39], individuals who present these types of extrinsic motivation are more likely to endorse sociocultural pressure towards thinness and to show more problematic eating behaviors and more psychological distress than individuals presenting more intrinsic motivation types. Our results are coherent with Pelletier and colleagues' work [39], since among women with higher depressive symptoms, the adoption of a more extrinsic motivation was related to more psychological distress, a lower self-esteem, more problematic eating behaviors (i.e., more disinhibition, more susceptibility to hunger), and a lower quality of life. Previous unsuccessful weight-loss attempts may have convinced these women that their efforts to regulate their eating behaviors had no influence on their life, or that results were depending on external forces not under their control, which would reinforce the extrinsic motivation.

Finally, it seems that Self-Determination, a personality trait, plays a central role in the psychological profile of overweight/obese women with higher depressive symptoms. Indeed, among these women, Self-Determination was linked to the majority of studied variables (depressive symptoms, disinhibition, susceptibility to hunger, self-esteem, body esteem, quality of life), whereas these links were not significant among women with lower depressive symptoms (except the link between Self-Determination and depressive symptoms). These results suggest that the incapacity to set goals for oneself and to take proper actions to achieve these goals, as well as the feeling of having no control over one's life, is crucial among women with higher depressive symptoms. It is also interesting to interpret this result in view of results regarding motivation. Indeed, it might be that since women with higher depressive symptoms are less competent in setting goals for themselves and have less confidence in their capacity to influence their life, they rely more on external sources than on their personal resources to motivate themselves. Besides, Self-Determination was negatively linked to introjected and external motivation to regulate eating behaviors. These women who reported being 


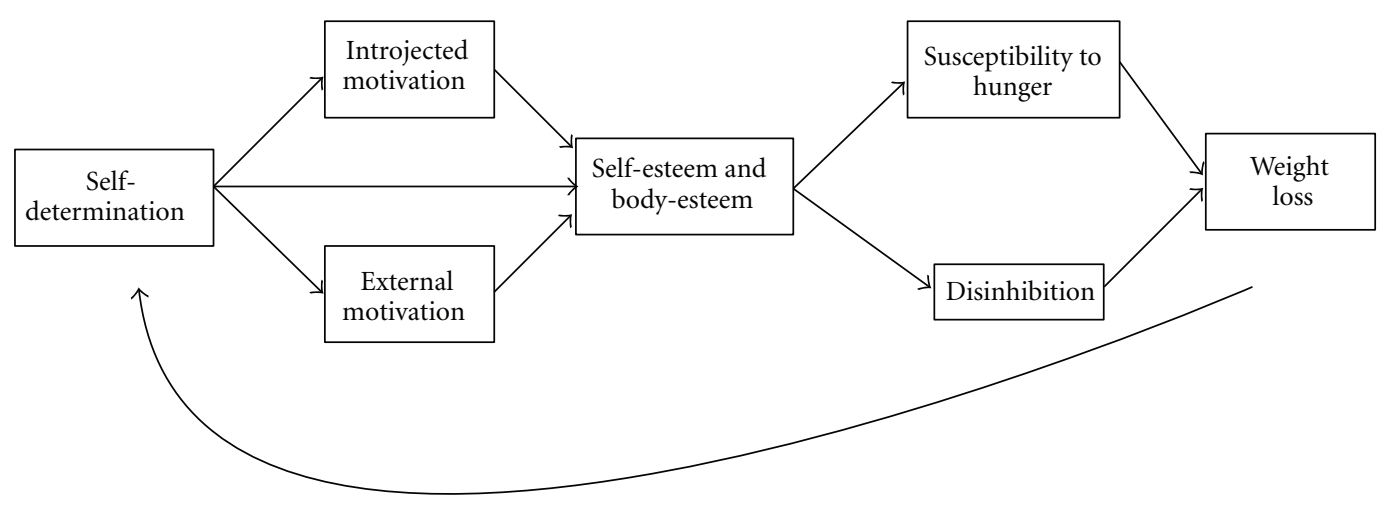

Depressive symptoms

FIGURE 1: Links between self-determination, motivation to regulate eating behaviors, self/body-esteem, eating behaviors, and weight loss.

less effective (Self-Determination) and presented a more extrinsic motivation also reported more negative self-esteem and body esteem, as well as a feeling of lacking control over eating (disinhibition and susceptibility to hunger), which could impede weight-loss attempts. In the end, the repetitive failures to lose weight might exacerbate the feelings of helplessness and negative self-perception that were already present. Figure 1 represents a model of the links between Self-Determination, motivation, self-perception, eating behaviors, and weight loss. Future studies will be needed to test this model in a larger sample.

However, this study also has important limitations. First, the sample size was relatively small, and after Bonferroni correction, most differences did not reach statistical significance. Yet, for the majority of these differences, effect sizes were moderate or large, suggesting that substantial differences existed between groups and could be evidenced in larger samples. Additional differences could also be evidenced with a larger sample. For example, an a posteriori power analysis revealed that both groups should have been formed of at least 40 participants in order to detect significant differences concerning motivation to regulate eating behaviors. Second, ecological validity was favoured in the present study, which means that internal validity was reduced in comparison to more controlled experimental designs. Since weight-loss methods were well diversified, the efficacy of the efforts undertaken by participants could not be measured, and their adherence to recommendations was not taken into account. Finally, according to the design of the study, it is not possible to infer causal relationships between studied variables. Future studies should explore the temporal links between these variables.

In conclusion, the presence of depressive symptoms, even if they are not clinically significant, is clearly an important marker of severity regarding several eating and psychological variables among overweight/obese women. The Beck Depression Inventory seems to be useful to identify this group of women who are more vulnerable to experience difficulties during weight-loss process.

\section{Acknowledgments}

We would like to thank Simone Lemieux for her careful revision of the manuscript

\section{References}

[1] World Health Organization, "Obesity: preventing and managing the global epidemic," Report of a WHO Consultation, Geneva, Switzerland, 2004, http://whqlibdoc.who.int/ trs/WHO_TRS_894.pdf.

[2] M. L. Fitzgibbon and D. S. Kirschenbaum, "Heterogeneity of clinical presentation among obese individuals seeking treatment," Addictive Behaviors, vol. 15, no. 3, pp. 291-295, 1990.

[3] M. A. Friedman and K. D. Brownell, "Psychological correlates of obesity: moving to the next research generation," Psychological Bulletin, vol. 117, no. 1, pp. 3-20, 1995.

[4] M. T. van der Merwe, "Psychological correlates of obesity in women," International Journal of Obesity, vol. 31, supplement 2, pp. S14-S18, 2007.

[5] R. A. Millstein, S. A. Carlson, J. E. Fulton et al., "Relationships between body size satisfaction and weight control practices among US adults," MedGenMed Medscape General Medicine, vol. 10, no. 5, article 119, 2008.

[6] L. A. Anderson, G. R. Janes, D. C. Ziemer, and L. S. Phillips, "Diabetes in urban African Americans, body image, satisfaction with size, and weight change attempts," The Diabetes Educator, vol. 23, no. 3, pp. 301-308, 1997.

[7] K. L. Green, R. Cameron, J. Polivy et al., "Weight dissatisfaction and weight loss attempts among Canadian adults," Canadian Medical Association Journal, vol. 157, supplement 1, pp. S17-S25, 1997.

[8] S. R. Davy, B. A. Benes, and J. A. Driskell, "Sex differences in dieting trends, eating habits, and nutrition beliefs of a group of Midwestern College students," Journal of the American Dietetic Association, vol. 106, no. 10, pp. 1673-1677, 2006. 
[9] M. Liebman, B. A. Cameron, D. K. Carson, D. M. Brown, and S. S. Meyer, "Dietary fat reduction behaviors in college students: relationship to dieting status, gender and key psychosocial variables," Appetite, vol. 36, no. 1, pp. 51-56, 2001.

[10] A. Blokstra, C. M. Burns, and J. C. Seidell, "Perception of weight status and dieting behaviour in Dutch men and women," International Journal of Obesity, vol. 23, no. 1, pp. 7-17, 1999.

[11] B. L. Wolfe and J. E. Smith, "Different strokes for different folks: why overweight men do not seek weight loss treatment," Eating Disorders, vol. 10, no. 2, pp. 115-124, 2002.

[12] K. Presnell, J. Pells, A. Stout, and G. Musante, "Sex differences in the relation of weight loss self-efficacy, binge eating, and depressive symptoms to weight loss success in a residential obesity treatment program," Eating Behaviors, vol. 9, no. 2, pp. 170-180, 2008.

[13] J. A. Linde, R. W. Jeffery, R. L. Levy et al., "Binge eating disorder, weight control self-efficacy, and depression in overweight men and women," International Journal of Obesity, vol. 28, no. 3, pp. 418-425, 2004.

[14] M. Heo, A. Pietrobelli, K. R. Fontaine, J. A. Sirey, and M. S. Faith, "Depressive mood and obesity in US adults: comparison and moderation by sex, age, and race," International Journal of Obesity, vol. 30, no. 3, pp. 513-519, 2006.

[15] C. Dong, L. E. Sanchez, and R. A. Price, "Relationship of obesity to depression: a family-based study," International Journal of Obesity, vol. 28, no. 6, pp. 790-795, 2004.

[16] A. F. Jorm, A. E. Korten, H. Christensen, P. A. Jacomb, B. Rodgers, and R. A. Parslow, "Association of obesity with anxiety, depression and emotional well-being: a community survey," Australian and New Zealand Journal of Public Health, vol. 27, no. 4, pp. 434-440, 2003.

[17] S. L. McElroy, R. Kotwal, S. Malhotra, E. B. Nelson, P. E. Keck Jr., and C. B. Nemeroff, "Are mood disorders and obesity related? A review for the mental health professional," Journal of Clinical Psychiatry, vol. 65, no. 5, pp. 634-651, 2004.

[18] R. E. Roberts, S. Deleger, W. J. Strawbridge, and G. A. Kaplan, "Prospective association between obesity and depression: evidence from the Alameda County study," International Journal of Obesity, vol. 27, no. 4, pp. 514-521, 2003.

[19] R. Rosmond, "Obesity and depression: same disease, different names?” Medical Hypotheses, vol. 62, no. 6, pp. 976-979, 2004.

[20] A. J. Stunkard, M. S. Faith, and K. C. Allison, "Depression and obesity," Biological Psychiatry, vol. 54, no. 3, pp. 330-337, 2003.

[21] F. S. Luppino, L. M. de Wit, P. F. Bouvy et al., "Overweight, obesity, and depression: a systematic review and meta-analysis of longitudinal studies," Archives of General Psychiatry, vol. 67, no. 3, pp. 220-229, 2010.

[22] E. Atlantis and M. Baker, "Obesity effects on depression: systematic review of epidemiological studies," International Journal of Obesity, vol. 32, no. 6, pp. 881-891, 2008.

[23] E. M. Venditti, R. R. Wing, J. M. Jakicic, B. A. Butler, and M. D. Marcus, "Weight cycling, psychological health, and binge eating in obese women," Journal of Consulting and Clinical Psychology, vol. 64, no. 2, pp. 400-405, 1996.

[24] M. Q. Werrij, S. Mulkens, H. J. Hospers, and A. Jansen, "Overweight and obesity: the significance of a depressed mood," Patient Education and Counseling, vol. 62, no. 1, pp. 126-131, 2006

[25] A. Jansen, R. Havermans, A. Roefs, and C. Nederkoorn, "Jolly fat or sad fat?: subtyping non-eating disordered overweight and obesity along an affect dimension," Appetite, vol. 51, no. 3, pp. 635-640, 2008.
[26] A. Jansen, A. Vanreyten, T. van Balveren, A. Roefs, C. Nederkoorn, and R. Havermans, "Negative affect and cue-induced overeating in non-eating disordered obesity," Appetite, vol. 51, no. 3, pp. 556-562, 2008.

[27] M. P. Gagnon-Girouard, C. Bégin, V. Provencher, A. Tremblay, S. Boivin, and S. Lemieux, "Subtyping weight-preoccupied overweight/obese women along restraint and negative affect," Appetite, vol. 55, no. 3, pp. 742-745, 2010.

[28] F. Bonnet, K. Irving, J. L. Terra, P. Nony, F. Berthezène, and P. Moulin, "Anxiety and depression are associated with unhealthy lifestyle in patients at risk of cardiovascular disease," Atherosclerosis, vol. 178, no. 2, pp. 339-344, 2005.

[29] A. N. Fabricatore, T. A. Wadden, R. H. Moore, M. L. Butryn, S. B. Heymsfield, and A. M. Nguyen, "Predictors of attrition and weight loss success: results from a randomized controlled trial," Behaviour Research and Therapy, vol. 47, no. 8, pp. 685691, 2009.

[30] R. A. Carels, H. M. Cacciapaglia, O. M. Douglass, S. Rydin, and W. H. O'Brien, "The early identification of poor treatment outcome in a women's weight loss program," Eating Behaviors, vol. 4, no. 3, pp. 265-282, 2003.

[31] S. D. Anton, C. K. Martin, L. Redman et al., "Psychosocial and behavioral pre-treatment predictors of weight loss outcomes," Eating and Weight Disorders, vol. 13, no. 1, pp. 30-37, 2008.

[32] E. Stice, K. Presnell, L. Groesz, and H. Shaw, "Effects of a weight maintenance diet on bulimic symptoms in adolescent girls: an experimental test of the dietary restraint theory," Health Psychology, vol. 24, no. 4, pp. 402-412, 2005.

[33] S. Sullivan, C. R. Cloninger, T. R. Przybeck, and S. Klein, "Personality characteristics in obesity and relationship with successful weight loss," International Journal of Obesity, vol. 31, no. 4, pp. 669-674, 2007.

[34] Airlie Consensus Conference, Standardization of Anthropometric Measurements, Human Kinetics, Champaign, Ill, USA, 1988.

[35] A. T. Beck, R. A. Steer, and G. K. Brown, BDI-II Manuel, Psychological Corporation, San Antonio, Tex, USa, 2nd edition, 1996.

[36] A. J. Stunkard and S. Messick, "The three-factor eating questionnaire to measure dietary restraint, disinhibition and hunger," Journal of Psychosomatic Research, vol. 29, no. 1, pp. 71-83, 1985.

[37] L. G. Pelletier, C. M. Blanchard, E. Sharp, N. Otis, and C. Amiot, "The Global Motivation Scale: a dispositional measure of intrinsic, extrinsic, and amotivationfor different life activities," Manuscript submitted for publication, University of Ottawa, 2002.

[38] E. L. Deci and R. M. Ryan, Intrinsic Motivation and SelfDetermination in Human Behavior, Plenum Press, New York, NY, USA, 1985.

[39] L. G. Pelletier, S. Dion, and C. Lévesque, "Can selfdetermination help protect women against sociocultural influences about body image and reduce their risk of experiencing bulimic symptoms?" Journal of Social and Clinical Psychology, vol. 23, no. 1, pp. 61-88, 2004.

[40] R. L. Kolotkin, S. Head, and A. Brookhart, "Construct validity of the impact of weight on quality of life questionnaire," Obesity Research, vol. 5, no. 5, pp. 434-441, 1997.

[41] R. L. Kolotkin and R. D. Crosby, "Psychometric evaluation of the impact of weight on quality of life-lite questionnaire (IWQOL-Lite) in a community sample," Quality of Life Research, vol. 11, no. 2, pp. 157-171, 2002. 
[42] B. K. Mendelson, M. J. Mendelson, and D. R. White, "Bodyesteem scale for adolescents and adults," Journal of Personality Assessment, vol. 76, no. 1, pp. 90-106, 2001.

[43] M. Rosenberg, Society and Adolescent Self-Image, Princeton University Press, Princeton, NJ, USA, 1965.

[44] A. Yarcheski and N. E. Mahon, "A causal model of positive health practices: the relationship between approach and replication," Nursing Research, vol. 38, no. 2, pp. 88-93, 1989.

[45] T. Damji, R. Clément, and K. A. Noels, "Acculturation mode, identity variation, and psychological adjustment," Journal of Social Psychology, vol. 136, no. 4, pp. 493-500, 1996.

[46] J. S. Fleming and B. E. Courtney, "The dimensionality of selfesteem: II. Hierarchical facet model for revised measurement scales," Journal of Personality and Social Psychology, vol. 46, no. 2, pp. 404-421, 1984.

[47] C. R. Cloninger, The Temperament and Character Inventory (TCI): A Guide to Its Development and Use, Washington University, Center for the Psychobiology of Personality, St. Louis, Mich, USA, 1994.

[48] C. R. Cloninger, "A systematic method for clinical description and classification of personality variants: a proposal," Archives of General Psychiatry, vol. 44, no. 6, pp. 573-588, 1987.

[49] J. Cohen, Statistical Power Analysis for the Behavioral Sciences, Lawrence Erlbaum Associates, New York, NY, USA, 2nd edition, 1988.

[50] P. Teixeira, S. B. Going, L. B. Houtkooper et al., "Weight loss readiness in middle-aged women: psychosocial predictors of success for behavioral weight reduction," Journal of Behavioral Medicine, vol. 25, no. 6, pp. 499-523, 2002.

[51] P. J. Teixeira, A. L. Palmeira, T. L. Branco et al., "Who will lose weight? A reexamination of predictors of weight loss in women," International Journal of Behavioral Nutrition and Physical Activity, vol. 1, article 12, 2004. 


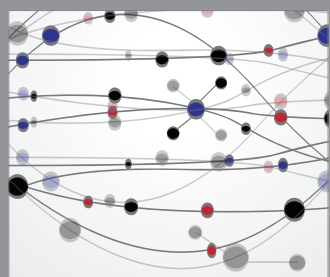

The Scientific World Journal
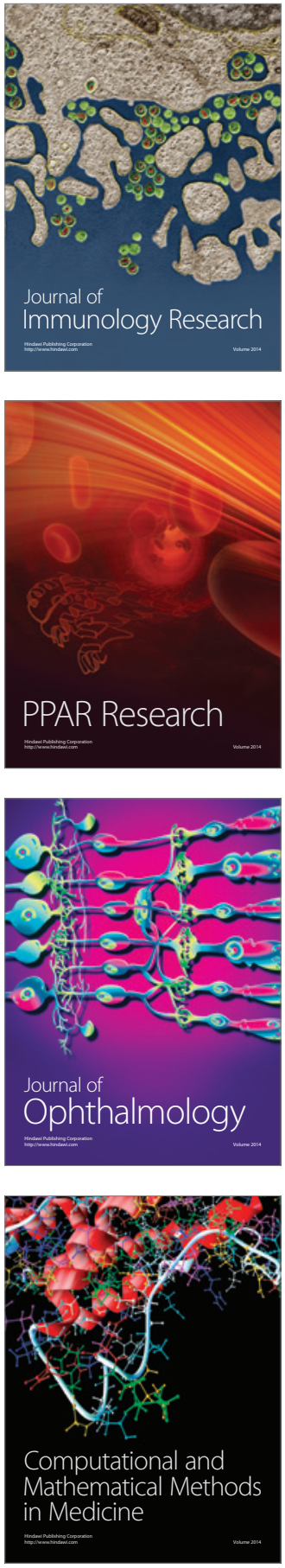

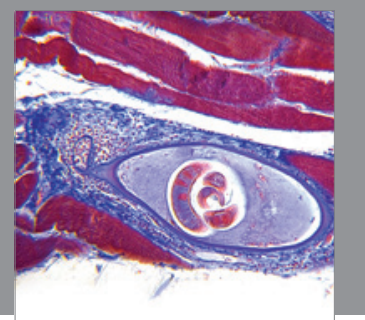

Gastroenterology

Research and Practice
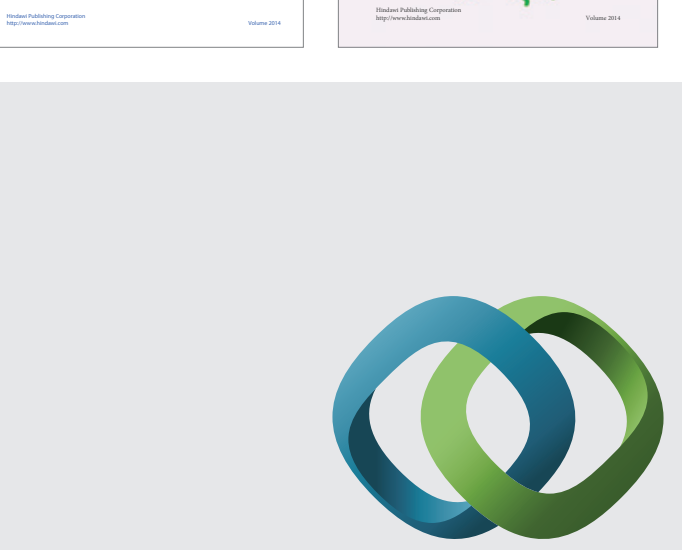

\section{Hindawi}

Submit your manuscripts at

http://www.hindawi.com
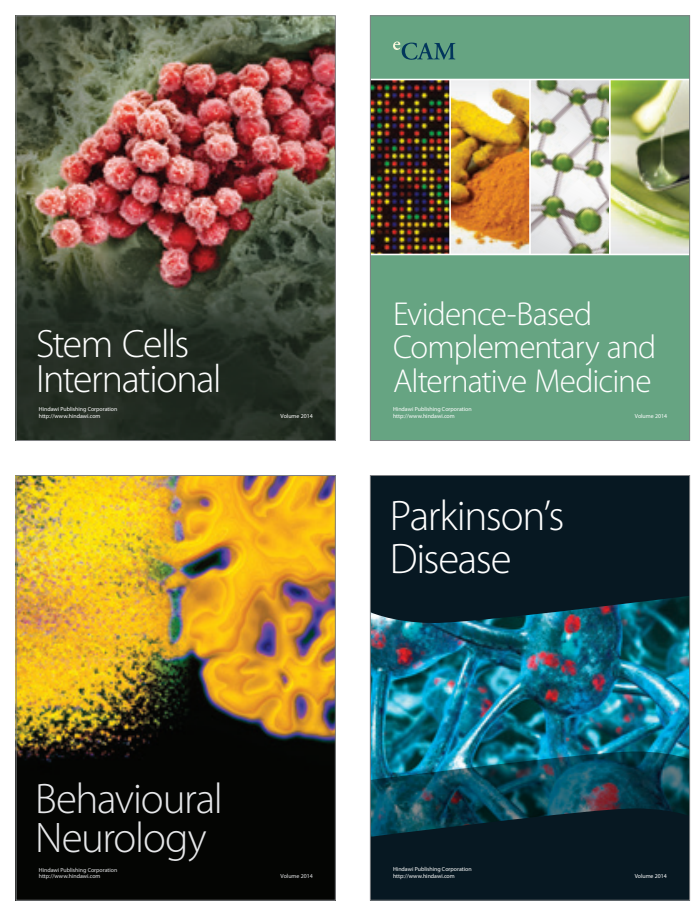

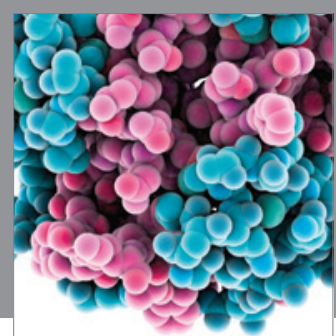

Journal of
Diabetes Research

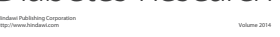

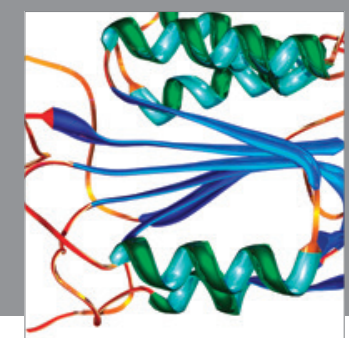

Disease Markers
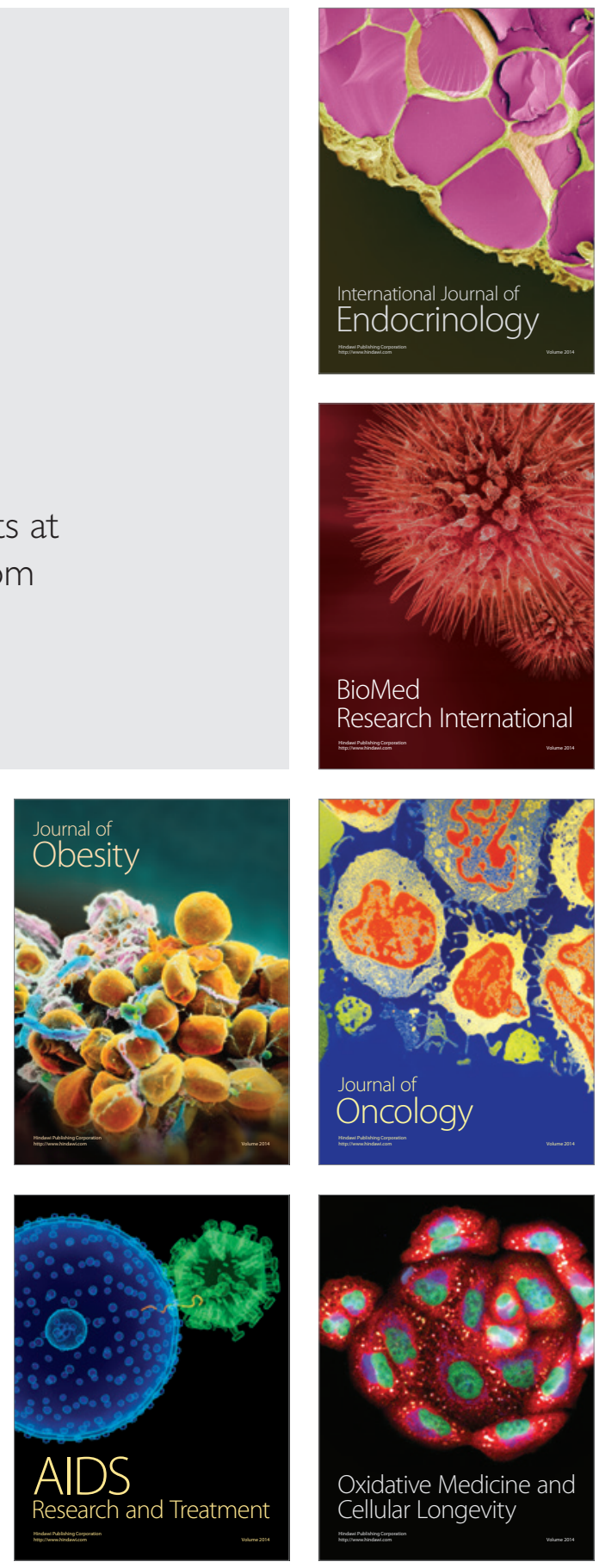